\title{
Ampliação do acesso à atenção oftalmológica: um estudo sobre a avaliabilidade da campanha “De Olho na Visão", Goiás, 2004
}

\author{
Evaluability assessment of a health care campagne for improving \\ the access to ophthalmologic care in a central region of Brazil, \\ 2004
}

Lazara Ribeiro Ferreira Lima $^{1}$

Ligia M aria Vieira daSilva²

\footnotetext{
${ }^{1}$ Secretaria deEstado da SaúdedeGoiás, Chefia Assessoria de Projetos Estratégicos. Rua SC 1 229, ParqueSanta Cruz. 74 860-260 Goiânia GO. lazara.lima@gmail.com ${ }^{2}$ Instituto deSaúde Coletiva, Universidade Federal da Bahia.
}

Abstract In order to identify the areas of priority for a systematic evaluation of a campaign for improving the access to ophthalmologic care "Keep an eye on your vision", occurred in the state of Goiás in 2004, an evaluability assessment was conducted by means of interviews with keyinformants and document analysis. A logical model was developed for providing dimensions and criteria for evaluation. This framework was submitted to a panel of experts using the Delphi method. The results revealed that the campaign was sufficiently structured to allow for systematic evaluations. Differences in the interpretation of the objectives and of the target population of the campaign between the experts wereverified. Problems related to accessibility and effectiveness indicated these components as priorities for evaluation. The authors discuss the relevance of evaluability assessment as a preliminary stage for the evaluation of programs.

Key words Evaluability, Evaluation, Campaign
Resumo Com o objetivo de identificar as áreas prioritárias para avaliação sistemática da Campanha "D eO Iho na V isão", voltada para a ampliação do acesso à atenção oftalmológica ocorrida no estado de Goiás em 2004, realizou-se um estudo sobre sua avaliabilidade por intermédio da realização de entrevistas com informantes-chave e de análise documental. Elaborou-se um model o lógico do qual foram derivados dimensões e critérios para avaliação. Submeteram-se esses critérios a um comitê de especialistas através da técnica de Delphi. Os resultados revelaram que a campanha encontrava-se estruturada o suficiente de modo a permitir a realização de avaliações sistemáticas a seu respeito. Verificou-se diversidade na compreensão acerca dos objetivos e da população-alvo, entre diferentes profissionais. Problemas relacionados com acessi bilidade e efetividade indicam essasdimensões como áreas prioritárias para avaliação. 0 estudo também funcionou como uma pré avaliação, gerando recomendações relacionadas com a logística. Os autores discutem a relevância da realização de estudos de avaliabilidade como etapa preliminar para a realização deavaliação de programas.

Palavras-chave Avaliabilidade, Avaliação, Campanha 
Introdução

A exemplo de outros estados brasileiros, Goiás vem assistindo, a partir da década de 1960, ao processo de envelhecimento de sua população relacionado, entre outras causas, ao aumento da expectativa de vida, à redução das taxas de fecundidade e de mortalidade. Em 2002, a população de 60 anos e mais do estado de Goiás apresentou crescimento de $65,2 \%$, em relação a 1993, superior àquele verificado na população total, que foi de $24 \%$ no período ${ }^{1}$. Esse fenômeno tem gerado novas demandas assistenciais, quer seja pela maior prevalência das doenças crônico-degenerativas no grupo de idosos ou pelas dificuldades de acesso dessa população às ações e serviços de saúde em decorrência das desigualdades regionais, sociais e de geração.

A catarata é considerada a maior causa de cegueira no mundo, atingindo em torno de 16 milhões de pessoas². No Brasil, existem aproximadamente 24,5 milhões de pessoas que apresentam algum tipo de incapacidade ou deficiência, ou seja, $14,5 \%$ da população. Estão incluídas nessa categoria pessoas com dificuldades de enxergar, de locomover ou que portam al gum tipo dedeficiênciafísica ou mental. A incapacidadede enxergar em seus diversos graus de severidade respondepor $48,1 \%$, do total mencionado eocupa o primeiro lugar. Da população portadora de algum tipo de dificuldade deenxergar, registrado na região Centro-Oeste, Goiás destaca-se concentrando mais de $40 \%$ da referida população ${ }^{1}$.

A catarata pode ser corrigida cirurgicamente. Relatos de cirurgias oculares bem-sucedidas têm sido relacionadas com a melhoria da qualidade devida deindivíduos dereduzido nível socioeconômico, entre 50 e 97 anos. A expectativa dos pacientes em relação à retomada de atividades após a cirurgia foi manifestada por $59,8 \%$ dos pacientes atendidos pelo Projeto Catarata ${ }^{3}$, em São Paulo, em 1999. No âmbito federal, desde 1999, a Campanha N acional de Cirurgias de Catarata foi desenvolvida pelo M inistério da Saúde em parceria com o Consel ho Brasileiro de O ftalmologia, tendo como objetivo eliminar a fila de pessoas cegas ou incapacitadas por catarata, no menor prazo possível 4 .

A rede assistencial do SUS, em Goiás, exibe baixa cobertura na oferta de serviços oftalmológicos - presentes em apenas dezesseis dos 246 municípios do estado - acarretando, conseqüentemente, uma significativa demanda reprimida. Em 2001, o estado de Goiás possuía 230 oftalmologistas para uma população de 3.093.171 habitantes, correspondente à densidade de um profissional para cada 18.412 habitantes ${ }^{5}$, oferta considerada ainda insuficiente para atendimento das necessidades populacionais. H avia uma concentração dos mesmos na cidade de Goiânia, em 2001, que contava então com 170 profissionais correspondentes a $74 \%$ do total dos oftalmologistas existentes no estado, estando os sessenta profissionais restantes distribuídos em vinte municípios ${ }^{5}$. Naquele contexto, a saúde ocular de pessoas idosas em Goiás vinha se constituindo em importante problema de saúde pública. Além disso, a grande extensão territorial do estado $\left(340.086 .698 \mathrm{~km}^{2}\right)$, ao lado da elevada proporção da população com renda menor que dois salários mínimos (60\%), constituíam-seem obstáculos adicionais ao acesso.

Para enfrentar esse quadro, a Secretaria de Estado da Saúde de Goiás realizou no ano de 2004 a segunda edição da Campanha "De O Iho na Visão", em parceria com o Centro de Referência em Oftalmologia do Hospital das Clínicas, da UniversidadeFederal de Goiás - CEROF/HC/ UFG -, com objetivo geral de ampliar 0 acesso à atenção oftalmológica no estado de Goiás.

Com a finalidade de subsidiar os gestores no processo de avaliação da campanha e visando a reunir informações para a tomada de decisões acerca da sua continuidade, al gumas questões de avaliação impunham-se. A primeira dizia respeito à avaliabilidade dessa campanha. Em que medida a intervenção realizada permite a realização de uma avaliação? Que problemas surgiram e quais as áreas críticas que requeriam avaliação? 0 presente estudo foi desenvolvido para buscar responder a algumas dessas questões visando a contribuir para formulação subseqüente de políticas na área de saúde ocular e apresenta parte dos resultados do referido estudo. 0 objetivo geral foi aferir a avaliabilidade da campanha "De O Iho na Visão" voltada para a ampliação do acesso à atenção oftalmológica no estado de Goiás em 2004. Os específicos foram: identificar problemas prioritários para avaliação da campanha e delimitar melhor os seus objetivos.

\section{M etodologia}

\section{Estratégia do estudo}

Realizou-se um estudo de caso da campanha. Os níveis de análise selecionados foram a gestão e as práticas assistenciais. Para cada nível de análise, foram selecionados dimensões, crité 
rios e indicadores a partir da elaboração da versão preliminar do modelo lógico da intervenção.

A avaliabilidade, anglicismo referente ao termo evaluability, que pode ser traduzido como "em que medida o programa pode ser avaliado", é o exame sistemático e preliminar de um programa, em sua teoria e em sua prática, a fim de determinar se há justificativa para uma avaliação extensa e (ou) para melhor delimitar os objetivos do programa, bem como identificar áreas críticas a serem priorizadas na avaliação ${ }^{6}$. Para merecer uma avaliação extensa, o programa deve operar como previsto, ser relativamente estável ao longo do tempo e indicar estar alcançando algum resultado positivo. Por outro lado, freqüentemente, os diversos gestores e profissionais responsáveis pela implementação do programa têm concepções distintas acerca dos objetivos do programa, assim como sobre aspectos mais frágeis que requerem avaliação ${ }^{6}$.
Modelo lógico, dimensões, critérios e indicadores

Com a finalidade de selecionar dimensões, critérios e indicadores para a pré-avaliação, dois modelos lógicos foram elaborados: um causal e outro relacionado com a intervenção propriamentedita. Para os dois níveis deanáliseescolhidos, derivaram-se do modelo preliminar as seguintes dimensões ecritérios: para o nível da gestão, as dimensões analisadas foram o planejamento e a avaliação. Em relação ao planejamento, os critérios propostos foram: a) existência de plano (clareza dos objetivos, coerência, factibilidade e estratégias); b) características do planejamento (estratégico, normativo); c) implementação e operacionalização do plano; d) coordenação (controle gerencial); e) supervisão e acompanhamento das ações (controle técnico). Já em relação à avaliação, os critérios selecionados, foram: a) existência desistema de informação (uso das informações, qualidade dos registros e das informações, banco de dados); b) logística (organização, triagem, encaminhamento, motivos das perdas e acessibilidade) (Quadro 1).

Quadro 1. Seleção de critérios para avaliação.

\begin{tabular}{|c|c|c|c|}
\hline Dimensão & Critérios & Indicadores & $\begin{array}{l}\text { Pontuação } \\
\text { (atribuir uma nota } \\
\text { de zero a dez) }\end{array}$ \\
\hline \multirow{6}{*}{ Gestão } & \multirow{5}{*}{ Planejamento } & $\begin{array}{l}\text { Existência de plano (clareza dos objetivos, } \\
\text { coerência, factibilidade e estratégias). }\end{array}$ & \\
\hline & & $\begin{array}{l}\text { Características do planejamento } \\
\text { (estratégico, normativo). }\end{array}$ & \\
\hline & & Implementação e operacionalização do plano & \\
\hline & & Coordenação (controle gerencial) & \\
\hline & & $\begin{array}{l}\text { Supervisão e acompanhamento das ações } \\
\text { (controle técnico) }\end{array}$ & \\
\hline & Avaliação & $\begin{array}{l}\text { Sistema de informação (uso das informações, } \\
\text { qualidade dos registros e das informações, } \\
\text { banco de dados) }\end{array}$ & \\
\hline Práticas & Logística & $\begin{array}{l}\text { Organização, triagem, encaminhamento, } \\
\text { motivos para as perdas e acessibilidade. }\end{array}$ & \\
\hline Sugestões & & & \\
\hline
\end{tabular}


Submeteu-se a matriz contendo dimensões, critérios e indicadores à apreciação do comitê de especialistas formado por quatro profissionais tanto da área acadêmica, quanto vinculados aos serviços de saúde. Para obtenção de consenso, utilizou-se a técnica de Delfos (D elphi $)^{7}$. Solicitou-se a cada membro do comitê que atribuísse uma nota de zero a dez, a cada um dos critérios (Tabela 1). Esclareceu-se que a nota zero corresponderia ao jul gamento de que o critério deveria ser excluído ea nota dez significaria que o especialista estaria atribuindo prioridade máxima ao mesmo. Os membros do painel poderiam sugerir novos critérios ou modificações àquel es apresentados. Encaminhou-se a matriz a cada membro do comitê via correio eletrônico, acompanhada de uma carta explicativa sobre o nome do pesquisador, sua instituição de trabalho, o obje tivo do estudo, a garantia do anonimato e do retorno dos resultados. Fez-sea consolidação dos dados por meio do cálculo das médias e desviospadrão. Devido ao elevado consenso, só foi efetuada uma rodada.

Técnicas de coleta e fontes de informação

As informações foram obtidas a partir de análise documental (fichas de inscrição, ficha de atendimento médico, fichas deencaminhamento do cliente para outras especialidades ou para retorno e manual de orientação para aplicação do teste de acuidade visual) e entrevista com informantes-chave.

Foram informantes-chave, num total denove entrevistados: a) técnicos de nível superior (mé dico, enfermeira, estatístico e coordenador da campanha); b) gestor municipal; c) usuários contemplados e não contemplados pela Campanha; d) agente comunitário de saúde - ACS. Obtevese o consentimento, informado por escrito, dos

Tabela 1 . Pontuação e média aritmética simples, segundo especialistas consultados sobre critérios para avaliação da campanha "De Olho na Visão". Goiás, 2004.

\begin{tabular}{|c|c|c|c|c|c|c|c|}
\hline \multirow{3}{*}{ Dimensão } & \multirow{3}{*}{ Critérios } & \multicolumn{6}{|c|}{ Pontuação (atribuir uma nota de zero a dez) } \\
\hline & & \multicolumn{6}{|c|}{ 1a rodada } \\
\hline & & $\begin{array}{c}\text { Especialista } \\
1\end{array}$ & $\begin{array}{c}\text { Especialista } \\
2\end{array}$ & $\begin{array}{l}\text { Especialista } \\
3\end{array}$ & $\begin{array}{c}\text { Especialista } \\
4\end{array}$ & M éd. & $\begin{array}{l}\text { Desvio } \\
\text { Padrão }\end{array}$ \\
\hline \multirow[t]{6}{*}{ Planejamento } & $\begin{array}{l}\text { Existência de plano (clareza dos } \\
\text { objetivos, coerência, factibilidade } \\
\text { e estratégias). }\end{array}$ & 10 & 9 & 10 & 10 & 9,7 & 0,5000 \\
\hline & $\begin{array}{l}\text { Características do planejamento } \\
\text { (estratégico, normativo) }\end{array}$ & 10 & 10 & 9 & 10 & 9,7 & 0,5000 \\
\hline & $\begin{array}{l}\text { Implementação / } \\
\text { Operacionalização do plano }\end{array}$ & 10 & 9 & 8 & 8 & 8,7 & 0,9574 \\
\hline & $\begin{array}{l}\text { Coordenação (controle } \\
\text { gerencial) }\end{array}$ & 8 & 10 & 9 & 10 & 9,2 & 0,9574 \\
\hline & $\begin{array}{l}\text { Supervisão / Acompanhamento } \\
\text { das ações (controle técnico) }\end{array}$ & 8 & 10 & 8 & 8 & 8,5 & 1,0000 \\
\hline & & 9 & 8 & 8 & 10 & 8,7 & 0,9574 \\
\hline Avaliação & $\begin{array}{l}\text { Sistema de informação (uso das } \\
\text { informações, qualidade dos } \\
\text { registros e das informações, } \\
\text { banco de dados) } \\
\text { Logística (triagem, organização, } \\
\text { encaminhamento, razão das } \\
\text { perdas eacessibilidade) }\end{array}$ & 10 & 9 & 9 & 10 & 9,2 & 0,9574 \\
\hline
\end{tabular}

Sugestões

Fonte: M atriz aplicada ao Comitêde Especialistas - nov./2004. 
entrevistados, tendo sido assegurado aos mesmos o sigilo econfidencialidade das informações por meio de protocolo previamente submetido a um comitê de ética. As entrevistas foram gravadas e em seguida foram transcritas na íntegra.

0 roteiro das entrevistas variou conforme as categorias de informantes. Os critérios ou variáveis adicionais indagados foram: objetivos; população-alvo; ad equação das estratégias utilizadas para 0 alcance dos objetivos propostos; aspectos já avaliados; organização do sistema de informação; problemas prioritários para a avaliação na opinião do entrevistado; usos dos resultados da avaliação; e problemas encontrados pelo entrevistado que merecem correção.

As respostas do comitê de especial istas foram consolidadas eas médias eo desvio-padrão, calculados. A partir dos critérios selecionados, as entrevistas foram analisadas, manualmente. Desta primeira leitura, os modelos lógicos foram reel aborados e aperfeiçoados. Fez-se a triangulação das informações obtidas por meio de uma comparação, critério a critério, entreas respostas dos diferentes informantes eentreestes eos documentos.

\section{Resultadosediscussão}

No que diz respeito tanto à definição dos objetivos da campanha, quanto ao que concerne à delimitação da população-alvo, verificou-se que, embora os mesmos estivessem claros para o coordenador da campanha, houve diversidade de opiniões entre informantes-chave entrevistados:

Os principais objetivos da campanha é o de levar atendimento oftalmológico a todas as cidades do Estado.

0 objetivo é detectar pessoas com problemas visuais, principalmente a catarata.

Contudo, pode-se deduzir, por meio do que foi efetivamente referido pelo agente comunitário de saúde-ACS e da entrevista com o coordenador, que o objetivo ampliou-se e passou a ser "atender às deficiências visuais detectadas na população com idade igual ou maior de 50 anos cadastradas pelas equipes do Programa de Saúde da Família-ESF". Porém, não fica claro em que medida essa falta de consenso implicou a operacionalização da campanha, indicando ser esta uma área problema a ser avaliada.

Quanto à logística, o processo de triagem da população pelos ACS mostrou-se uma estraté gia adequada, pois os mesmos conhecem as necessidades de saúde da população do território quese encontra sob seu domínio. No entanto, os próprios ACS entrevistados referem ao pouco tempo para divulgar a campanha, o que pode ter resultado nas baixas coberturas encontradas. Aqui se indaga: será que toda a população que necessita do atendimento foi contatada? Soube da campanha? Em relação ao encaminhamento do paciente para o reexame, observa-se que 0 fluxo está bem definido, porém, problemas relacionados com a acessibilidade foram apontados:

É do tumulto, da espera, porque as pessoas idosas não sabem da dificuldade, né?Então eles não esperam, acabam desistindo. E às vezes, assim, eu tenho caso na minha área de pessoas que fizeram a triagem, detectamos o problemas, aí eles demoraram eessa pessoa procurou outro atendimento, outro lugar.

U ma coisa que desestimula a genteéo desinteresse de al guns prefei tos e secretários de saúde. Isso aí, foi o que desestimulou muito. A gente chega lá para atender, tem uma cidadequeé 12.000 pacientes, ele não leva um paciente ea gente vê e tá bem claro que é política, problemas políticos, que são adversários políticos, sei lá, al guma coisa tá interferindo naquilo ali.

As entrevistas revelam, portanto, uma perda de pacientes triados quenão compareceram para o reexame, cabendo indagar: qual a magnitude deste não comparecimento? Q uais as razões? Estes problemas de acessibilidade foram generalizados? Qual a cobertura real da campanha?

Quatro áreas comportam necessidades de avaliação da campanha em nível de suas práticas: a) no processo de triagem pelos ACS; b) no atendimento clínico (reexame); c) na seleção para recebimento de óculos; d) na recuperação da visão dos usuários.

O resultado da pontuação atribuída pelo comitêde especialistas aos critérios prioritários para avaliação, com base no cálculo das médias e do desvio-padrão, encontra-se expresso na Tabela 1. Os dados encontrados mostram que a existência de plano foi considerada pel os especial istas como um dos principais critérios para avaliação da campanha $(9,7)$; verificou-se ainda elevado consenso em relação à cobertura e eqüidade $(9,5)$. As características do planejamento, implementação, coordenação ea logística da campanha ocupam o terceiro lugar $(9,0)$; 0 sistema de informação $(8,7)$ e, por último, o critério com menor pontuação foi a existência de supervisão/acompanhamento $(8,2)$.

Em síntese, o presente estudo revelou que a campanha "De Olho na Visão" encontra-se estruturada o suficiente deforma a viabilizar a realização de avaliações sistemáticas a seu respeito. 


\section{Considerações finais erecomendações}

0 esforço desenvolvido neste estudo para aferir a avaliabilidade da campanha "De Olho na Visão", do estado de Goiás, atingiu seus objetivos no sentido de identificar possibilidades e apontar áreas prioritárias para a avaliação.

Diantedosachados do presenteestudo, podese formular algumas considerações e recomendações para subsidiar os gestores no aperfeiçoamento da estratégia aqui analisada:

1. A existência de uma demanda não atendi- da na campanha, de pessoas com o diagnóstico visual, porém, para as quais não houve distribuição de óculos, requer a identificação deestratégias que respondam a essas necessidades;

2. A acessibilidade, por intermédio da revisão da logística da campanha, poderá ser ampliada.

O bserva-se que esses resultados são também consistentes com outros estudos que destacam as importantes contribuições da avaliabilidade como uma etapa da avaliação, além de ajudar o avaliador a potencializar sua capacidade produtiva, seus insights e julgamentos de valor ${ }^{8}$.

\section{Colaboradores}

LRF Lima participou da concepção, pesquisa, metodologia e redação do texto. Silva LM V participou da revisão crítica e aprovação da versão a ser publicada.

\section{Referências}

1. Instituto Brasileiro de Geografia e Estatística (IBGE). Rio de Janeiro: IBGE; 2000.

2. Schwab L. Cataract. In: Schwab L. Eye care in developing nations. $3^{\text {rd }}$ ed. San Francisco: The Foundation of the American Academy of Ophthalmology; 1999. p. 21-52.

3. Kara-Júnior N, Temporini ER, Kara-José N. Cataract surgery: expectations of patients assisted during a community project in São Paulo, state of São Paulo, Brazil. Rev. Hosp. Clín. Fac. Med. S. Paulo 2001; 56(6):163-168.

4. Brasil. Ministério da Saúde. Assistência de média e alta complexidade no SUS- 1995/2000/ Secretaria de Assistência à Saúde. Brasília: M inistério da Saúde; 2001.

5. Conselho Brasileiro de Oftalmologia. Censo 2001: distribuição dos oftalmologistas por estado. São Paulo: CBO; 2001.

6. Rossi PH, Freeman HE. Evaluation - a systematic approach. Thousand Oaks: Sage Publications; 1999.

7. Donabedian A. An introduction to quality assurance in health care. Oxford: Oxford University Press; 2003.

8. Leviton LC, Collins CB, Laird BL. Teaching Evaluation U sing Evaluability Assessment. Evaluation 1998; 4(4):389-409.

Artigo apresentado em 05/07/2006

Aprovado em 26/06/2007

Versão final apresentada em 07/08/2007 